\title{
An Advanced Architecture \& Instrumentation for Developing the System of Monitoring a Vital Sign (Oxygen Saturation) of a Patient.
}

\author{
${ }^{1}$ Md.Mokarrom Hossain, ${ }^{2}$ A.S.M.Mohsin*, ${ }^{3}$ Md.Nasimul Islam Maruf, ${ }^{4}$ Md. Asaduzzaman Shoeb* \\ and ${ }^{5}$ Ifat Al-Baqee
}

\begin{abstract}
Bio-Medical Engineering (BME) has been an active research area for years. BME is the application of engineering techniques to the medical fields. It combines the design and problem solving skills of engineering with medical sciences to improve the health care and the quality of individual life. With the proliferation of advanced electronics and applied engineering, the ways to help people specifically patients are changed. Therefore, an advanced design for monitoring a vital sign towards experimentation is presented. The vital signs monitor is essentially a real time device that displays certain received signals from the human body. The projects of this sort under taken so far have used windows to display the output whereas this project aims to generate a real time display of the signals received from the human body using Linux. Linux was preferred for its ability to simultaneously process data and display it in real time. The basic principles and methods are outlined. The major components of the system are shown separately, and are discussed individually. Few electronic devices used for data are depicted and described. The final results show a better performance
\end{abstract}

Index Terms-Oximeter, Data Acquisition Card, Pulse Oximetry, Design Process Overview, Result Analysis

\section{INTRODUCTION}

The objective of Patient Monitoring Systems is to have a quantitative assessment of the important physiological variable of patients during critical periods of biological functions. For diagnostic and research purposes it is necessary to know actual value or trend of change of this variable. Patient monitoring systems are used for measuring continuously or at regular intervals, automatically, the values of patient's important physiological parameters.

There are several categories of patients who may need continuous monitoring or intensive care. Critically ill patients recovering from surgery, heart attack or serious

Manuscript Received September 29, 2010

${ }^{1}$ Md.Mokarrom Hossain, ${ }^{2}$ A.S.M.Mohsin, ${ }^{3}$ Md. Nasimul Islam Maruf , ${ }^{4} \mathrm{Md}$. Asaduzzaman Shoeb, ${ }^{5}$ Ifat Al-Baqee, is with(1-5), Department of Electrical and Electronic Engineering, Stamford University Bangladesh,51Siddeswari, Dhaka1217,Bangladesh..

* Member of IACSIT (email: ${ }^{1}$ mokarromnhossain@gmail.com, ${ }^{2}$ asmmohsin@gmail.com, ${ }^{3}$ mrf5 9bd@yahoo.com,shoeb_1064@yahoo.com, ${ }^{5}$ ifty00@gmail.com) illness, are often placed in special units, generally known As intensive care units, where they can be supervised constantly by the use of electronic instruments. The main features of patient monitoring systems:

- Organizing and displaying information in a meaningful form to improve patient care.

- Correlating multiple parameters for clear demonstration of clinical problems.

- Processing the data to set alarms on the development of abnormal conditions.

- Ensuring better care.

The most often monitored biological functions are Electrocardiogram (ECG), heart rate, pulse rate, temperature and respiratory rate. Among this oxygen saturation is the centre of this thesis.

\section{Pulse Oximeter, Oxygen Saturation $\left(\mathrm{SpO}_{2}\right)$}

Oximeter is based on the concept that arterial oxygen saturation determinations can be made using two wavelengths. The two wavelengths assume that only two absorbers are present, ox hemoglobin and reduced hemoglobin. Light passing through the finger will be absorbed by skin pigments, tissue, cartilage, bone, arterial blood, venous blood but this absorption is indifferent to the wavelength of the light being passed through it. Therefore the absorbance by all these helps us in the determination of oxygen saturation in the blood.

\section{DATA ACQUiSITION CARD}

The Data Acquisition Card (DAQ card) was used to digitize the analog output of the circuits. Certain changes were made to the circuits to accommodate this process. The DAQ card fed its digitized data to the computer which worked using Linux. This ensured real time display as the aim of this project was to enhance the real time display characteristics of a Vital Signs Monitor.

\section{A. Medical background}

Pulse oximetry is a non-invasive and continuous method of determining the amount of oxygenated and deoxygenated hemoglobin in a person's blood supply. It is preferable to have a direct measure of the oxygen levels in hemoglobin because it can be determined in real time while causing no discomfort to 
the patient. Traditional pulse oximeters take measurements from the finger and ear lobe.

\section{B. Mathematical Aspect}

Pulse oximetry is accomplished by implementing the Beer-Lambert Law, which, in this case, relates the concentration of oxygen in the blood to the amount of light absorbed when transmitted through the blood. The absorption of the light transmitted through the medium can be calculated using the Beer-Lambert Law as follows:

$$
\mathbf{I}_{\text {OUT }}=\mathbf{I}_{\text {IN }} \mathrm{e}^{-\mathrm{A}}
$$

Where $\mathrm{I}_{\mathrm{OUT}}$ is the intensity of the light transmitted through the medium, $\mathrm{I}_{\mathrm{IN}}$ is the intensity of the light going into the medium, and $\mathrm{A}$ is the absorption factor.

\section{A brief history of oximetry}

To be able to design a pulse oximeter the theory behind how the body absorbs oxygen and how the absorption of light works must be clear. The knowledge that is used is that saturated hemoglobin is light red and non-saturated hemoglobin is dark red.

The first pulse ox meter was designed in the late 1930's by German researchers whose objective was to measure the oxygenation of "high altitude pilots."[6] From that point on, the pulse ox meter, as it was later called, has been improved upon continuously.

The first pulse oximetry was invented in 1972 and has grown to become an increasingly important technique in Intensive Care Units and medical care in general. It is a simple non-invasive method of monitoring the percentage of hemoglobin $(\mathrm{Hb})$, which is saturated with oxygen.

\section{Principles of Pulse Oximetry}

The purpose of a pulse oximeter is to measure the percentage of hemoglobin, which is saturated with oxygen. The theory behind the technique is based on the red and infrared (IR) light absorption characteristics of oxygenated and deoxygenated hemoglobin. Oxygenated hemoglobin absorbs more infrared light and allows more red lights to pass through. For deoxygenated hemoglobin it is the other way around. Deoxygenated hemoglobin absorbs more red lights and allows more infrared light to pass through. Red light is in the $600-750 \mathrm{~nm}$ wavelength light band. Infrared light is in the 850-1000 nm wavelength light band. Figurel shows the absorption coefficient for both types of hemoglobin at different wavelengths.

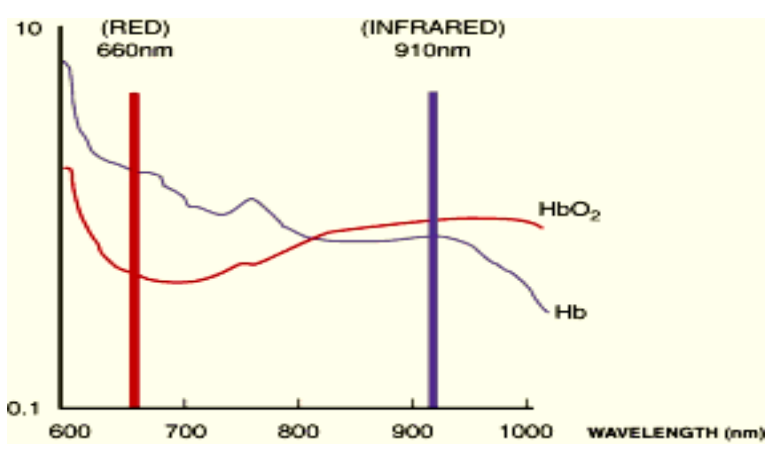

Fig1: The absorption coefficient for the two types of hemoglobin as a function of wavelength.

Pulse oximetry uses two light emitting diodes (LEDs) or light sources of similar kind with known wavelengths, one LED emitting red light and the other infrared.

There are two methods of sending light through the measuring site:

- Transmission and

- Reflectance.

In the transmission method, a photo detector detects the light that passes through.

In the reflectance method, it is also possible to have the photo detector placed right next to the LEDs and have it receiving the reflected light.

This method is not as common. The transmission method is the most common type used and for this discussion the transmission method will be implied.
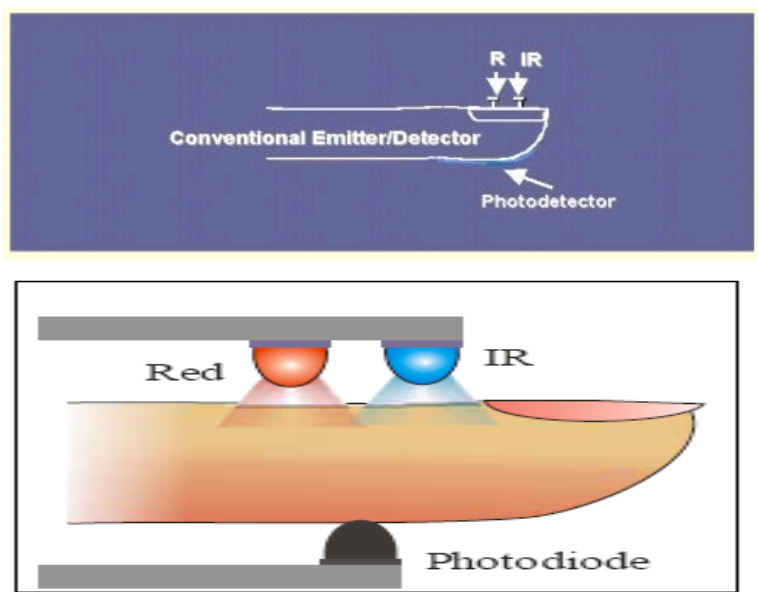

Fig 2: Basic idea of a pulse oximeter of haemoglobin at red and infrared wavelength.

Two signals will be generated by the photodetector, one signal created by the red light, and another signal created by the IR light. The signals will differ from one another because of the difference in light-absorption discussed above. The more oxygen there is in the blood, the more the two signals will differ. Obviously, the tissue will absorb light as well, but since the tissues' absorption coefficient is identical for both wavelengths, it will not affect the result.

Absorption on each wavelength differs significantly for the oxyhaemoglobin and deoxygenated haemoglobin. Therefore 
from the difference of the absorption of the red and infrared light the ratio between oxy/deoxyhaemoglobin can be calculated. As the amount of blood in the capillaries depends on the actual blood pressure, which varies around the heart, the heart pulse cycle and in turn the heart rate can also be measured.

Oxy hemoglobin refers to oxygen carrying haemoglobin and deoxygenated haemoglobin refers to non oxygen carrying haemoglobin. If all haemoglobin molecules bonded with an oxygen molecule $\left(\mathrm{O}_{2}\right)$, the total body of haemoglobin is said to be fully saturated $(100 \%$ saturation). When haemoglobin unloads the oxygen molecule to tissue cells at capillary levels, the saturation progressively decreases and the normal venous saturation is about $75 \%$. The normal saturation level is said to be between $87-97 \%$.

The two wavelengths are chosen because deoxygenated haemoglobin has a higher absorption at around $660 \mathrm{~nm}$ and at $910 \mathrm{~nm}$ oxygenated hemoglobin has the higher absorption.

The measurement Process: The measurements taken by the pulse oximeter demonstrate the shape of a pulsatile waveform as seen in fig3. This pulsatile waveform has both AC and DC components in it. The DC components are comprised of the absorption from the non-pulsing arterial blood, the venous and capillary blood, as well as from scattering and absorption due to the tissue and bone. These components are always constant and rest on one another as shown in the figure. The AC component of the figure 4 is the pulsatile waveform that we are interested in.

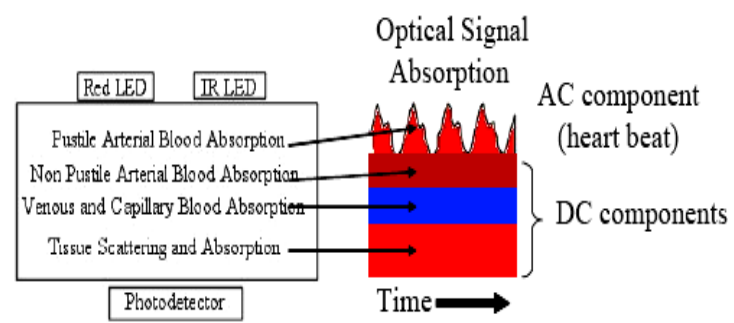

Fig 3: AC and DC components of oximetry

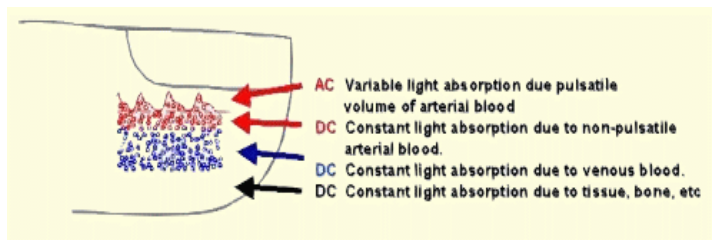

Fig4: AC and DC components of oximetry

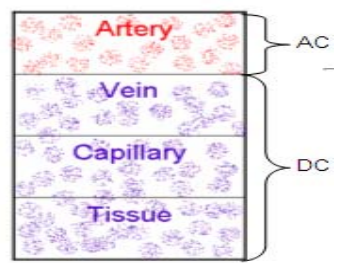

Fig 5: AC and DC components of oximetry

This waveform represents the pulsing of the blood in the arteries and each individual pulse can be seen, representative of the heart rate. This waveform is gathered for both light frequencies, in this case infrared and red light. In order to obtain the pulse oximeter saturation (Sp02), these AC and DC components from each of the wavelengths need to be measured and taken as a ratio as follows:

$$
\mathrm{R}=[\mathrm{AC} \lambda 1 / \mathrm{DC} \lambda 1] /[\mathrm{AC} \lambda 2 / \mathrm{DC} \lambda 2]
$$

$$
\text { Ratio, } \begin{aligned}
& R=\frac{(A C / D C)_{r}}{(A C / D C)_{i}} \\
& \text { SPO} 2=(110-25 \mathrm{R}) \%
\end{aligned}
$$

\section{E. Calibration}

This ratio is then used in a calibration curve based on studies of healthy individuals to determine the $\mathrm{Sp}_{2}$. This value will end up being a percentage which will tell the physician whether or not everything is as it is supposed to be. A normal saturation level is between $87-97 \%$. This method of measuring the $\mathrm{Sp}_{2}$ has been shown to be accurate to within $2.5 \%$.

\section{F. Oxygen Saturation Circuit requirements}

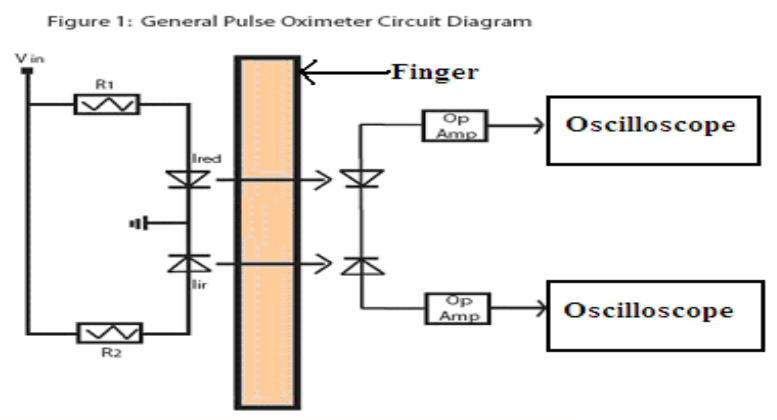

Fig 6: General Pulse Oximeter Circuit Diagram

The sensor is to be made out of a black rubber-made tube. It is important to use a material that does not reflect so much light and does not let light through the surface. The front of the tube was tightened with a black plug to avoid surrounding light will affect the result. Two holes are drilled in the top part of the tube, for the two diodes $(660 \mathrm{~nm}$ and $940 \mathrm{~nm})$ and two holes in the bottom part for the Photodiode. The electronics are set up on a lab-board. It's built by connecting the Photodiode of the sensor to an amplification circuit.

The following instruments have been used to power the electronics and to measure the result:

- 1 multimeter Fluke 45 dual display multimeter

- 1 oscilloscope HAMEG 60 MHz HM604

- 1 power supply D2510.

\section{G. Design Process Overview}

Due to the dearth of sensors in the market, it was required to 
attempt reverse engineering to construct a signal that will be similar to that expected to be received by the circuit. Under this objective, the circuits required can be broken down into certain parts.

\section{Desian Flowchart}

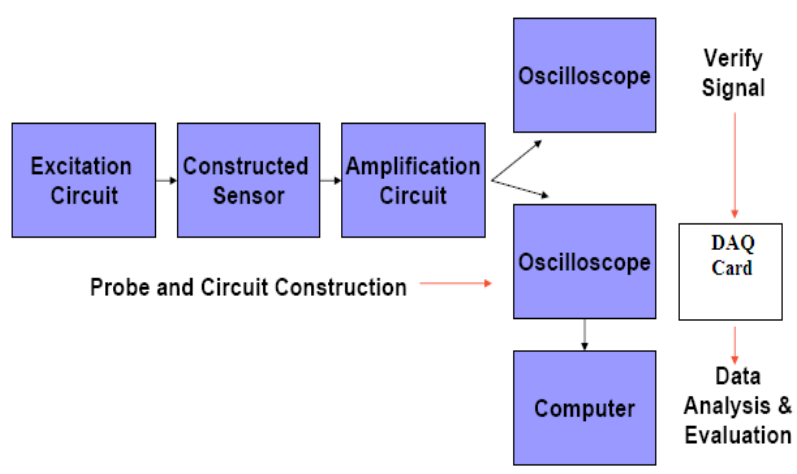

Fig 7: Flow Chart

\section{H. Complete Probe and Circuitry Prototype Construction}

The pulse oximeter design consists of LED excitatory circuits as well as photodiode sensory circuits; the receiving circuits convert the red and infrared light currents into voltages which can then be observed using an oscilloscope. Signal voltage data is then recorded and post-processed in excel in order to obtain blood oxygen saturation levels. The approach can be broken down as follows:

1) Two light emitting diodes (LEDs), of red (600 to $750 \mathrm{~nm})$ and infrared $(940 \mathrm{~nm})$ wavelengths were directed at tissue and activated by an excitatory circuit.

2) The light emitted from the LEDs were transmitted through the skin and detected by two photodiodes. An infrared rejection filter photodiode was then placed across from the red LED in order to detect transmitted red light and prevent infrared light interference. Similarly, a visible light rejection filter photodiode was placed across from the infrared LED with similar intentions.

3) The two photodiodes were then connected to a trans impedance amplification circuit that converted the current to an appropriatelyenhanced voltage signal.

\section{Amplification}

The pulsating changes in transmitted light through the patient are very weak, and so the analog signal output of the frequency to voltage stage is also very small in amplitude. Therefore, a high gain amplifier stage is required to provide a suitable waveform. The high gain of the circuit requires that it also be immune to environmentally induced noise.

Infrared Photodiode Transimpedance Amplification Circuit
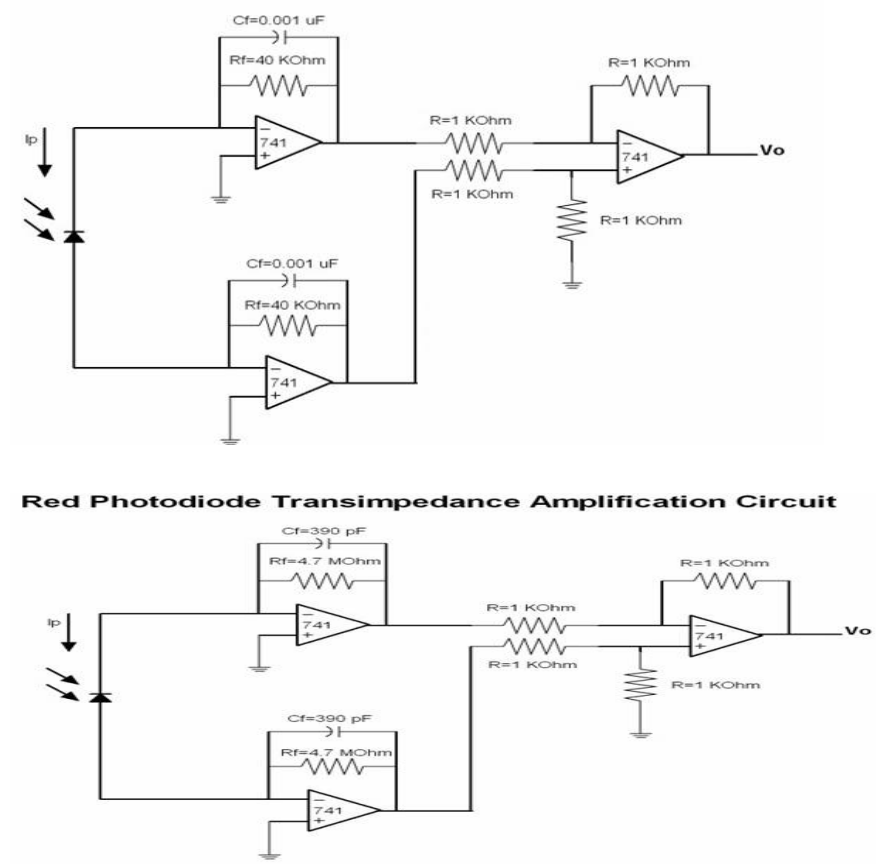

Fig 8 and Fig 9: Trans impedance Amplification Circuit

The above two circuits were implemented. Red and Infrared emitters and detectors were implemented into separate circuits

\section{EXPERIMENTAL DATA OF OXYGEN SATURATION}

\begin{tabular}{|l|l|}
\hline Thickness & Output(v) \\
\hline 1 & 0.517 \\
\hline 2 & 0.515 \\
\hline 3 & 0.51 \\
\hline 4 & 0.509 \\
\hline 5 & 0.508 \\
\hline 6 & 0.507 \\
\hline 7 & 0.506 \\
\hline 8 & 0.506 \\
\hline 9 & 0.505 \\
\hline 10 & 0.505 \\
\hline
\end{tabular}

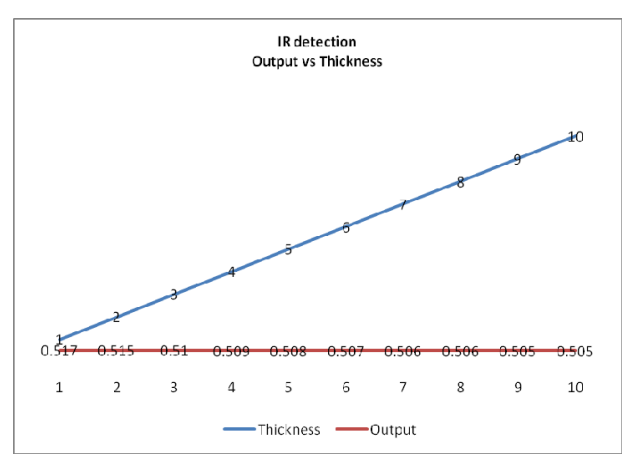


Fig 10: Thickness vs. Output Voltage

\section{A. Result Analysis}

Unfortunately, the devices available in the market here are not appropriate for the circuit. Hence proper output was never recorded in this channel. However, we conducted reading based on paper thickness and tabulated it. A positive correlation was observed in this data analysis.

\section{CONCLUSION}

In this project we tried to ameliorate the overall performance of a oxygen saturation monitoring system. The entire architecture is implemented which successfully reads output through the DAQ card and displays it in soft real time. The purpose of the project was to enhance the real time properties of the display of human physiological variables and that has been successfully implemented and recorded.

To extend its application, it will be possible to use the same channel and feed the input into the DAQ card which in turn will provide the digitized input to the Linux computer. Real time Linux can be used in this project without any further modification the input into the DAQ card which in turn will provide the digitized input to the Linux computer.

Real time Linux can be used in this project without any further modification.

\section{REFERENCES}

[1] Body Temperature, Bets http://health.yahoo.com/infectiousdisease-symptoms/body temperature/healthwise

[2] Md. Mazlan Abdul http://www.geocities.com/nazlen_2000/web

[3] Applications for Pico Products, Pico Technology Limited, St. Neots, Cambridgeshire, UK, "Electrocardiogram (ECG) project for DrDaq," 2005

[4] http://www.picotech.com/applications/ecg.html.

[5] http://focus.ti.com/docs/solution/folders/print/330.html

[6] http://www.vision.net.au/ apaterson/science/hypoxia.htm

[7] http://www.faqs.org/docs/Linux-HOWTO/RTLinuxHOWTO.html\#s3

[8] http://focus.ti.com/docs/solution/folders/print/330.html

[9] E. Hill, M.D. Stoneham, "Practical Applications of Pulse Oximetry", World Federation of Societies of Anaesthesiologists, Issue 11, Article 4, 2000.

[10] Biomedical Instrumentation and Measurements,Prentice-Hall.

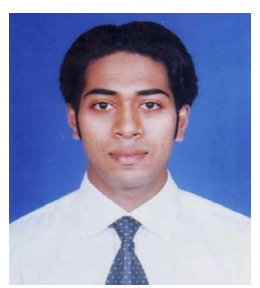

Md. Mokarrom Hossain born in Dhaka, Bangladesh on 31st dec, 1987.Gradutated on November, 2008 in Electrical \& Electronic Engineering from Islamic University of Technology, Dhaka, Bangladesh. The degree named as Bachelor of Science in Electrical \& Electronic Engineering. After finishing his graduation he joined as a lecturer in a private university named IBAIS University, Dhaka. During his period of teaching he engaged himself in extensive research works and projects which lead him to publish several journals in various renowned national and international journals. As a matter of progress he joined Stamford University Bangladesh where he got to know several other contemporary young researchers who were also working in various projects. That helped him to boost up his research relative activities a lot. Now with the help of his colleagues he is continuously working to improve the existing technology related to the field of Biomedical Engineering. E-mail: mokarromnhossain@gmail.com

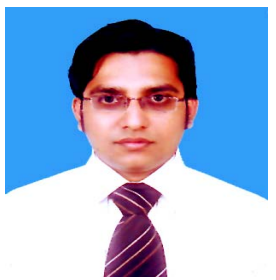

A.S.M.Mohsin was born in Comilla, Bangladesh in 1987.He received his B.Sc. in Electrical and Electronic Engineering from the Islamic University of Technology, Bangladesh in November 2008. He has been working as a Lecturer at the Department of Electrical and Electronic Engineering of Stamford University Bangladesh, since 2009. He is a member of IACSIT. His conducted courses in the undergraduate level are Optoelectronics, Power System Protection, Electronics, and Microwave Engineering etc. His research interests include Nanotechnology/Photonics, Renewable Energy, Bio-medical Engineering, and Communication. He has around 13 (International 6, National 7) published journal \& 2 international conference paper in distinguished journals \& conferences in the field of Renewable Energy, Nanotechnology ,Biomedical Engineering\& Image Processing. E-mail: asmmohsin@gmail.com

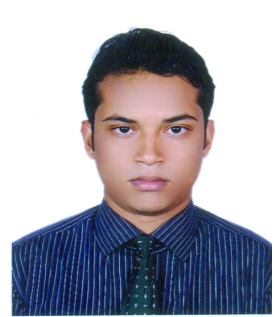

Md. Nasimul Islam Maruf was born in Dhaka, Bangladesh in 1986. He completed his B.Sc. in Electrical and Electronic Engineering from the Islamic University of Technology, Bangladesh in November 2008. He has been working as a Lecturer at the Department of Electrical and Electronic Engineering of Stamford University Bangladesh, Bangladesh since June 2009. His conducted courses in the undergraduate level are Power System I, Power Plant Engineering, Digital Signal Processing, Control Systems Lab, Power Electronics Lab etc. His research interests include Renewable Energy, Bio-medical Engineering, FACTS and Control Systems.E-mail: mrf59bd@yahoo.com

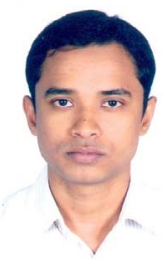

shoeb_1064@yahoo.com
Md. Asaduzzaman Shoeb was born in Barisal, Bangladesh. He was graduated in Electrical and Electronic Engineering from Islamic University of Technology (IUT), Dhaka in 2008. He is a lecturer at EEE Department of Stamford University Bangladesh. He has a very diverse research interest including renewable energy, biomedical engineering, data communication E-mail: shoeb1064@stamforduniversity.edu.bd, and control system. He is a member of IACSIT.

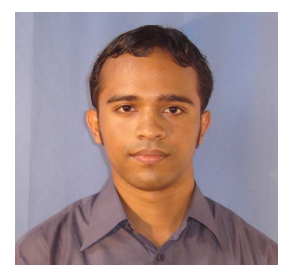

Ifat-Al-Baqee is a Lecturer at Department of Electrical and Electronic Engineering of Stamford University Bangladesh at Siddeswari, Dhaka, Bangladesh. He teaches electromagnetism and electronics. His research activities are related to Image Processing and Biomedical Engineering. Lecturer Baqee is graduated from Military Institute of Science and Technology, Dhaka, Bangladesh. He is a co-author of an international journal paper, "A Proposed Design for the Development of Vital Sign (Electrocardiogram) Patient Monitoring System", International Journal of Engineering Science and Technology (IJEST) ISSN: 0975-5472 (online version), Vol. 2, Issue No. 10, page 5813-5817, 2010.E-mail: 5ifty00@gmail.com 